\title{
Traffic Measurement of Traffic Light Based on Binocular Vision
}

\author{
Yue Wu, Linli Zhou and Yue Duan ${ }^{+}$ \\ Institute of Intelligent Machines, Chinese Academy of Sciences, Hefei 230031, China
}

\begin{abstract}
The accidents of the traffic light intersection have posed a great threat to the safety of people's life and property. In this paper, we study a kind of the front of the traffic light distance measuring technology based on binocular vision, through two CCD cameras capture the image information in front of traffic lights from different perspectives, we get two pieces of binocular images at the left and right, which are processed , positioned, and then, we find the matching points of the corresponding feature with using a global matching method of feature point extracted, which obtain parallax, then according to the principle of triangulation again, we restore the three-dimensional information of the measured object and depth of information to achieve the distance in front of traffic lights measurement. The experimental simulation results show that the design can effectively measure the distance in front of the traffic light, simple and effective, and has the high accuracy of measurement.
\end{abstract}

Keywords: binocular vision, camera calibration, stereo matching, three-dimensional distance measurement

\section{Introduction}

With the vigorous development of China's automobile industry and transportation industry, intelligent in the car safety driving system has become increasingly important position. The problem of target recognition and distance measurement based on binocular vision has always been the focus and difficult problem in the research and development of automobile safety driving system. It is based on image processing, covering the pattern recognition, computer technology, signal processing technology, electronic technology and automation control technology and other disciplines of cross technology. In recent years, domestic and foreign experts and scholars made in-depth research, and achieved some results in binocular stereoscopic vision ranging.

At present, the photoelectric distance measurement technology can be divided into active ranging and passive ranging. In the measurement process, the active ranging needs to use the light source to the measured object to apply energy. And then through detecting and analyzing of the reflected waves or the characteristics of the light to calculate the distance measured object [1]. The use of active ranging methods are generally ultrasonic, infrared, laser ranging and so on. Passive ranging using natural light reflection, through the image acquisition device to the target object for image acquisition, according to a certain geometric features and related principles of the image processing and analysis, to achieve the target object distance measurement. Photoelectric passive ranging technology is mainly based on binocular stereo vision ranging, based on different angles measured ranging and other methods. As the photoelectric passive ranging with no need to send any signal, does not touch the measured object and does not affect the performance of the measured object and shape characteristics, therefore, photoelectric passive ranging has the advantages of good concealment, repeatable measurement, simple structure and easy operation. At the same time, it has the advantages of fast measurement speed, simple structure, convenient operation and high measurement precision. Therefore, the distance measurement technology based on binocular stereoscopic should be widely

+ Corresponding author. Tel.: + 15256951533 .

E-mail address: wuyue@iim.ac.cn. 
used in military and life. This paper studies the application of parallel binocular vision ranging technology to the measurement of the distance of the front traffic light. The experimental results show that the design of the system is simple and fast, and the measurement accuracy is high, which can effectively realize the measurement of the distance of the front traffic light. The research work has laid a good theoretical foundation for the intelligent and production of automobile safety driving system.

\section{System Process Design}

In this paper, the flow of the front traffic light distance measurement system based on binocular vision includes the left and right CCD camera image acquisition, image processing, traffic light positioning, template matching and distance calculation and other components, the system process shown in Figure 1.

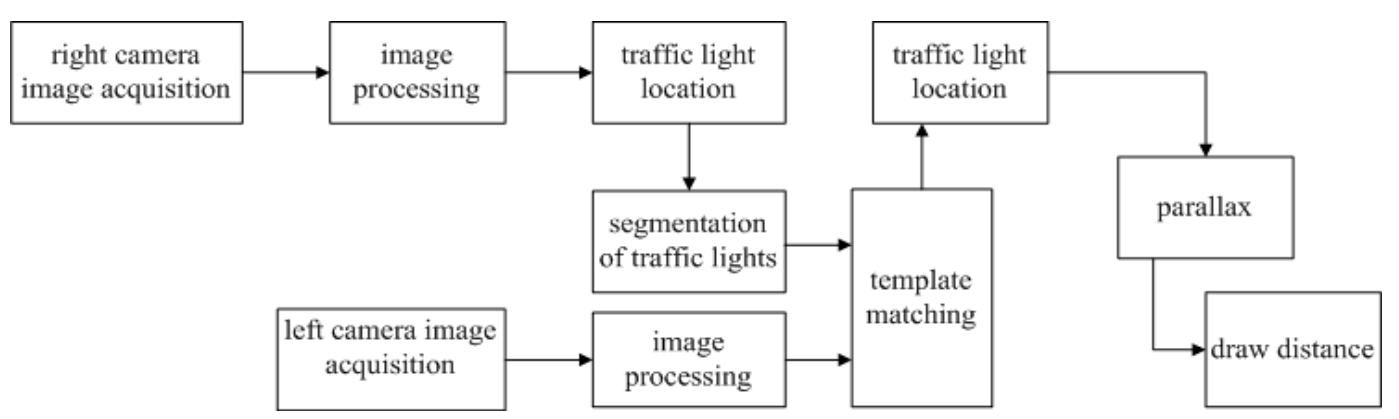

Fig. 1: Flow chart of front traffic light distance measurement system based on binocular vision.

When the measurement system is started, it is initialized first. With the left and right CCD camera in parallel position, the traffic lights are collected from the front of the different viewing angles. The system takes the image sequence from the collected image sequence in order. Due to the lack of noise and lack of light, the image is processed. After the gray scale processing algorithm is used to grays the left and right images, and then the left and right images are smoothed, corroded and opened and closed, and so on. The detailed features and contrast of the images are improved, and the calibration results of the inside and outside parameters of the camera are calculated. Then the right camera to capture the right image of the traffic light positioning, split the location of the traffic light after the image, the use of template matching method and the image after the left camera to capture the left image matching, and then use the maximum cross-correlation method to get the match image. Finally, the feature points are extracted from the upper left vertex of the rectangular image of the right image, and the corresponding matching points are found on the left graph by the global matching method. The pixel coordinates of the same point in the positioning image and the matching image are obtained, The According to the internal and external parameters of the camera and the triangulation principle, the three-dimensional information and depth information of the measured object are obtained by using the imaging difference of the same target on different projection images. The distance between the front traffic lights can be obtained.

\section{Binocular Vision Ranging System}

Binocular stereoscopic vision also known as stereoscopic vision. Binocular stereoscopic threedimensional distance measurement by imitating people's eyes to obtain the three-dimensional information of a target object in the surrounding environment. People 's eyes from a little bit different from the two angles to observe the same object will certainly be different, it is because people are between the eyes of the distance. Specifically, when the observer observes the same pixel, there will be different positions in the retina of the left and right eyes, and the difference in visibility is called binocular parallax. Binocular vision uses two cameras instead of the human eye to reconstruct the three-dimensional information of the object based on the principle of parallax, obtain the object depth information, and then get the distance. The system uses the most classic binocular binocular stereoscopic vision, which is composed of exactly the same two parallel placed CCD camera, the geometric correspondence shown in Fig 2. Let $M$ be a point on the measured object, the combined optical path focal length is $f^{\prime}$, the camera to the measured object depth distance is $\mathrm{S}$. 


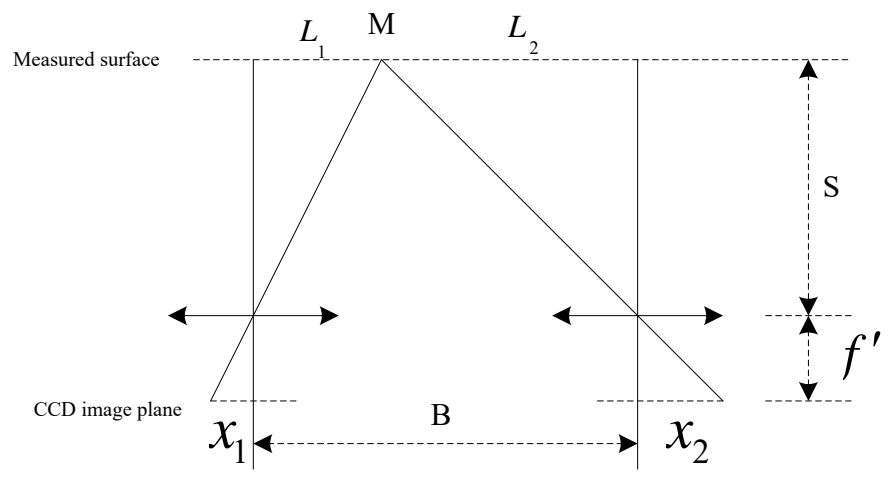

Fig. 2: Parallel binocular CCD parallax distance diagram.

Two parallel-placed camera machines take the same target point $M$ from different directions to obtain the left and right two-dimensional images. The target point $\mathrm{M}$ is imaged in the $x_{1}$ and $x_{2}$ positions of the two images respectively. From the triangular similarity principle, the formula is as follows.

$$
\left\{\begin{array}{c}
\frac{L_{1}}{x_{1}}=\frac{S}{f^{\prime}} \\
\frac{L_{2}}{x_{2}}=\frac{S}{f^{\prime}} \\
B=L_{1}+L_{2}
\end{array}\right.
$$

Since $x=x_{1}+x_{2}$, then the distance from the camera to the measured object point is:

$$
S=\frac{B f^{\prime}}{s}
$$

In the case where the arm length B and the combined optical path focal length $f^{\prime}$ are determined, the measured distance $\mathrm{S}$ and the difference $x$ of the binocular optical imaging position are inversely proportional to each other, and as long as the binocular optical path imaging position difference $x$ is obtained, the measured distance $\mathrm{S}$.

\section{Binocular Vision Camera Calibration}

In binocular stereoscopic rangefinder, the parameters that need to be calibrated by the camera are internal parameters and external parameters, which are based on the relationship between the pixel position of the binocular camera image and the location of the scene by using the camera model. From the image coordinates and world coordinates of the feature points obtained earlier, the internal and external parameters of the camera model are obtained [7].

\subsection{Reference Coordinate System and Correlation}

In the binocular stereoscopic disparity ranging, the image transformation is described by the imaging transformation. There are three different reference coordinate systems in the process of transformation: the image coordinate system (including the image pixel coordinates and the imaging plane coordinate system), the world coordinate system and the camera coordinate system [8]. The following definition of the transition between the different reference standard lines, as shown in Fig 3.

The physical dimensions of each pixel in the $\mathrm{X}$-axis and $\mathrm{Y}$-axis directions are DX and DY, and the coordinate relationship between the image pixel coordinate system and the imaging plane coordinate system of the image coordinate system is: 


$$
\left\{\begin{array}{l}
u=\frac{X}{d X}+u_{0} \\
v=\frac{Y}{d Y}+v_{0}
\end{array}\right.
$$

The conversion between the world coordinate system and the camera coordinate system is shown in Fig 4.

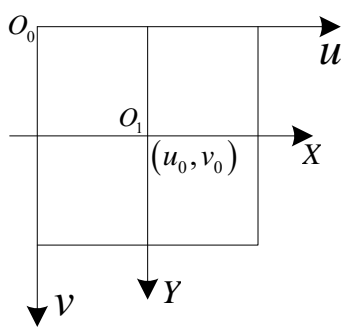

Fig. 3: Image coordinate system.

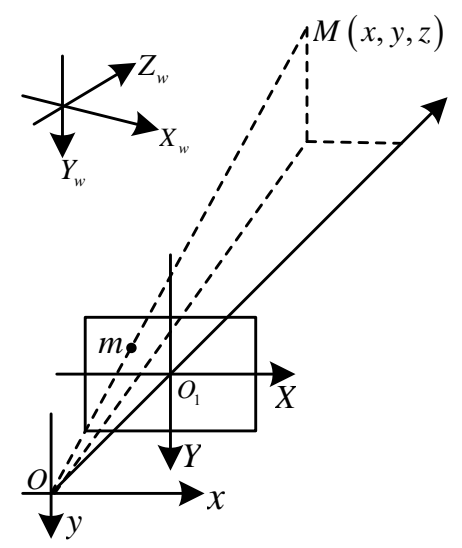

Fig. 4: Relationship between camera coordinate system and world coordinate system.

The coordinates of the world at a point $\mathrm{M}$ in space are $X_{w}=\left(X_{w}, Y_{w}, Z_{w}, 1\right)^{T}$. The coordinates of the camera coordinate system are $x=(x, y, z, 1)^{T}$. Where $\mathrm{R}$ is a $3 * 3$ orthogonal matrix, $\mathrm{t}$ is the three-dimensional translation vector, $0=\left(\begin{array}{lll}0 & 0 & 0\end{array}\right)^{T}, \mathrm{M}$ is a $4 * 4$ matrix, and the existence of the relationship is:

$$
\left\lfloor\begin{array}{l}
x \\
y \\
z \\
1
\end{array}\right\rfloor=\left[\begin{array}{ll}
R & t \\
0^{T} & 1
\end{array}\right]\left\lfloor\begin{array}{l}
X_{w} \\
Y_{w} \\
Z_{w} \\
1
\end{array}\right\rfloor=M\left\lfloor\begin{array}{l}
X_{w} \\
Y_{w} \\
Z_{w} \\
1
\end{array}\right\rfloor
$$

\subsection{Camera Model}

The system uses a classic pinhole imaging camera model, that is, any point $\mathrm{M}$ in the image projection position $\mathrm{m}$ is the optical center $\mathrm{O}$ and $\mathrm{M}$ point of the connection $\mathrm{OM}$ and the intersection of the plane, this relationship is called perspective projection or center Projection. The above-mentioned perspective projection relationship [7] is given by matrix and homogeneous coordinates:

$$
\eta\left[\begin{array}{c}
u \\
v \\
1
\end{array}\right]=\left[\begin{array}{ccc}
\frac{1}{d X} & 0 & u_{0} \\
0 & \frac{1}{d Y} & v_{0} \\
0 & 0 & 1
\end{array}\right]\left[\begin{array}{cccc}
f & 0 & 0 & 0 \\
0 & f & 0 & 0 \\
0 & 0 & 1 & 0
\end{array}\right]\left[\begin{array}{cc}
R & t \\
0^{T} & 1
\end{array}\right]\left[\begin{array}{c}
X_{w} \\
Y_{w} \\
Z_{w} \\
1
\end{array}\right]
$$




$$
=\left[\begin{array}{cccc}
\alpha_{x} & 0 & u_{0} & 0 \\
0 & \alpha_{y} & v_{0} & 0 \\
0 & 0 & 1 & 0
\end{array}\right]\left[\begin{array}{cc}
R & t \\
0^{T} & 1
\end{array}\right]\left\lfloor\begin{array}{l}
X_{w} \\
Y_{w} \\
Z_{w} \\
1
\end{array}\right\rfloor=M_{1} M_{2} X_{w}=M X_{w}
$$

where $\eta$ is a scale factor, $\left(\begin{array}{lll}u & v & 1\end{array}\right)$ is the projection homogeneous coordinate of point $\mathrm{M}$ in the image coordinate system, the rotation matrix is $\mathrm{R}$, the translation vector is $\mathrm{t}, \alpha_{x}=f / d X$ is the normalized u-axis focal length, $\alpha_{y}=f / d Y$ is the normalized v-axis focal length, $\mathrm{M}$ is the projection matrix of $3 * 3$, $\alpha_{x}, \alpha_{y}, u_{0}, v_{0}$ determine $M_{1}$, but also constitute the internal parameters of the camera, the world coordinate system direction and position determines the $M_{2}$, which id called the camera outside the parameters, the camera's internal and external parameters can be obtained, called the camera calibration ${ }^{[2]}$.

\subsection{Camera Calibration}

3D stereo target camera calibration method can be applied to any camera model, but the calculation process is complex, the cost of making calibration equipment is high, and subject to certain processing accuracy. In this paper, we use the method of camera calibration based on 2D planar target proposed by Zhang [6]. Which is based on the pinhole imaging model to determine the internal and external parameters of the camera. The correspondence between the target point in the spatial coordinate system and its image points on the image plane is:

$$
\eta \tilde{m}=A\left[\begin{array}{ll}
R & t
\end{array}\right] \tilde{M}
$$

The corresponding homogeneous coordinates are $\tilde{M}=[x, y, z, 1]^{T} \quad \tilde{m}=[u, v, 1]^{T}$

The camera's internal parameter matrix is:

$$
A=\left\lfloor\begin{array}{ccc}
\alpha_{x} & r & u_{0} \\
0 & \alpha_{y} & v_{0} \\
0 & 0 & 1
\end{array}\right\rfloor
$$

There is a transformation matrix between the target point and the corresponding image point, therefore:

$$
\eta \tilde{m}=H \tilde{M}
$$

where, $H=\lambda A\left[\begin{array}{ll}R & t\end{array}\right], h=\left[\begin{array}{lll}h_{1} & h_{2} & h_{3}\end{array}\right]$ is a $3 * 3$ matrix, $\lambda$ is a constant factor, remember $H=\left[\begin{array}{lll}h_{1} & h_{2} & h_{3}\end{array}\right]$, there are:

From the above formula can be drawn:

$$
\left[\begin{array}{lll}
h_{1} & h_{2} & h_{3}
\end{array}\right]=\lambda A\left[\begin{array}{lll}
r_{1} & r_{2} & t
\end{array}\right]
$$

$$
\left\{\begin{array}{c}
h_{1}^{T} A^{-T} A^{-1} h_{2}=0 \\
h_{1}{ }^{T} A^{-T} A^{-1} h_{1}=h_{2}{ }^{T} A^{-T} A^{-1} h_{2}
\end{array}\right.
$$

Using Cholesky matrix decomposition algorithm, we can get:

$$
\begin{aligned}
& r_{1}=\lambda A^{-1} h_{1}, \quad r_{2}=\lambda A^{-1} h_{2} \\
& r_{3}=r_{1} * r_{2}, \quad t=\lambda A^{-1} h_{3} \\
& \lambda=1 /\left\|A^{-1} h_{1}\right\|=1 /\left\|A^{-1} h_{2}\right\|
\end{aligned}
$$

The above principle is the principle of $2 \mathrm{D}$ plane target method to determine the parameters inside and outside the camera. In practical applications [4], the binocular stereoscopic range system is calibrated to the left and right cameras with a standard calibration template to obtain the rotation matrix between the two cameras after calibration and the R-shift vector $t$. We can achieve the camera inside and outside the parameters of the calibration. 


\section{Application of Binocular Vision in Ranging}

In this paper, the binocular vision ranging system is used to measure the distance of the traffic lights in front, and the image is collected, processed and positioned by the front traffic lights. Then, the parallax of the feature points is solved by stereo matching [5]. According to the principle of triangulation, the measurement of the distance between the front traffic lights is realized.

\subsection{Simulation of Binocular Vision Ranging}

First of all, the left and right binocular CCD camera in parallel position, from different angles to collect the front traffic light image, the system will be collected from the sequence of images taken out of a frame image, as shown in Fig 5.

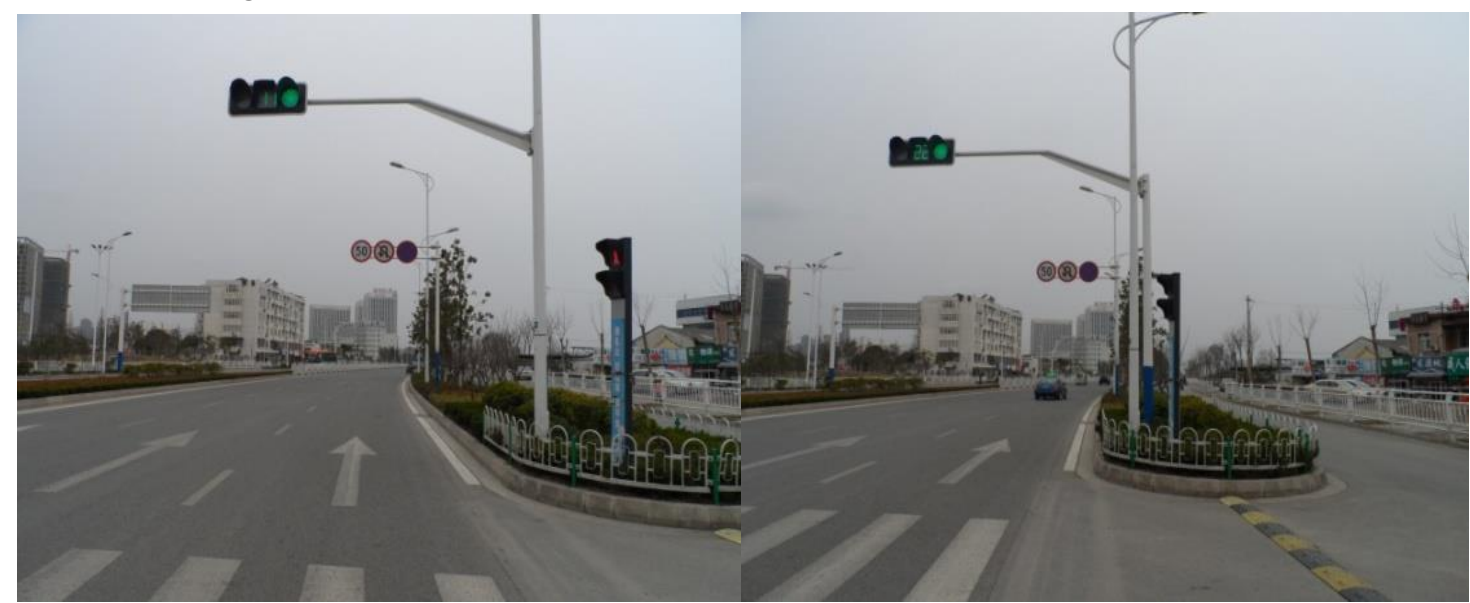

(a) Right side of the original image of the traffic light

(b) Left side of the original image of the traffic light

Fig. 5: The binocular CCD camera captures the left and right original images.

Next, the right image collected by the right camera is processed. Corresponding to the traffic lights, traffic lights are positioned according to the characteristics of bright, marked with the rectangular box, red light and image segmentation after positioning, as shown in Fig 6.
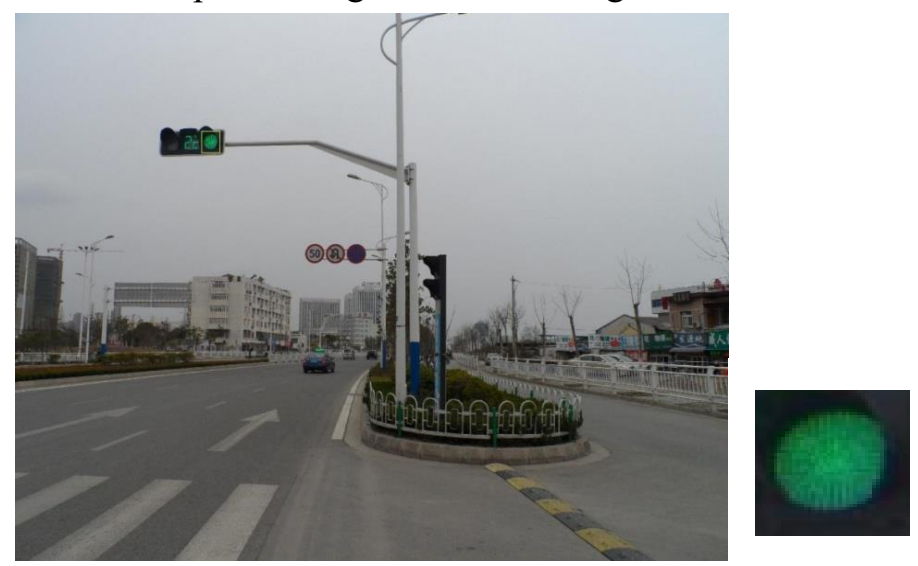

(a) The positioning image of the right traffic light (b)The segmented positioning image of the right traffic light

Fig. 6: The positioning and segmented image of the right traffic light.

Then, using the template matching method, match the red light positioning image segmented on the right with the left image after processing collected by the camera, and then use the maximum cross-correlation method to get the matching image, marked with a rectangular box, as shown in Fig 7.

Finally, the feature points are extracted from the upper left vertex of the rectangular image of the right image, and the corresponding matching points are found by the global matching method. The pixel coordinates of the same feature points in the positioning image and the matching image are obtained. And then according to the calculated camera internal and external parameters and triangulation principle, using the same target in different projection images on the imaging differences and then restore the measured 
target of three-dimensional information and depth information to achieve the measurement of the front of the traffic light distance.

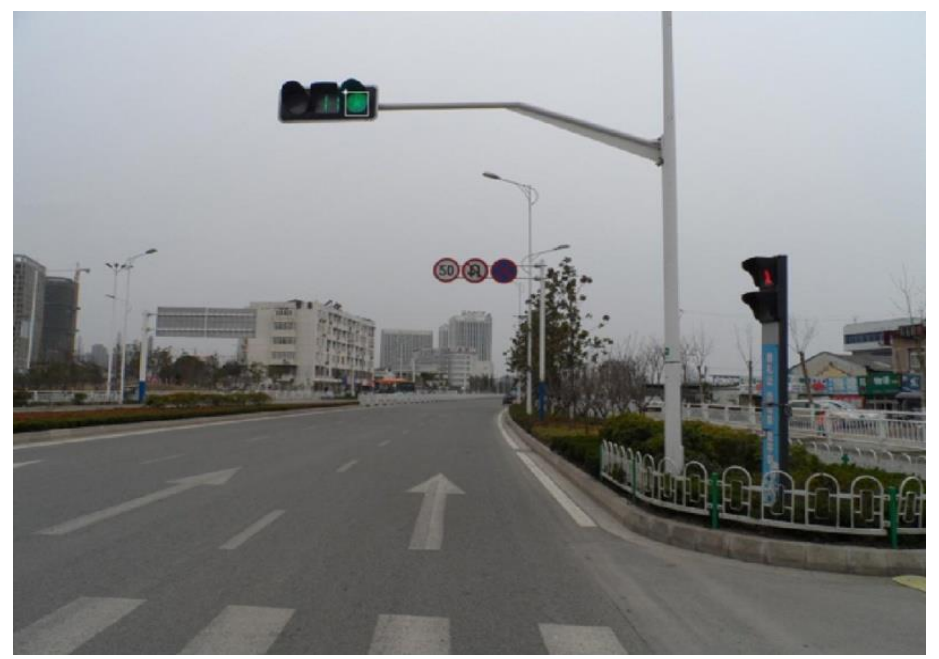

Fig. 7: The matching image of the right side of the traffic image in the left image.

\subsection{Analysis of Results}

In this experiment, we use the parallel position of the left and right binocular CCD cameras to collect the front traffic light images from different angles at different positions away from the front traffic lights. The experimental data are shown in Table 1.

Table 1: Front Traffic Light Distance Measurement Results

\begin{tabular}{|c|c|c|c|c|}
\hline id & $\begin{array}{c}\text { Measuring distance } \\
/ \mathrm{m}\end{array}$ & $\begin{array}{c}\text { Actual distance } \\
/ \mathrm{m}\end{array}$ & $\begin{array}{c}\text { Deviation } \\
/ \mathrm{m}\end{array}$ & Relative error \\
\hline $\mathbf{1}$ & 7.62 & 8.00 & -0.38 & $4.75 \%$ \\
\hline $\mathbf{2}$ & 9.66 & 10.00 & -0.34 & $3.40 \%$ \\
\hline $\mathbf{3}$ & 11.29 & 12.00 & -0.71 & $5.08 \%$ \\
\hline $\mathbf{4}$ & 15.38 & 15.00 & 0.38 & $2.50 \%$ \\
\hline $\mathbf{5}$ & 19.04 & 20.00 & -0.96 & $4.80 \%$ \\
\hline $\mathbf{6}$ & 26.23 & 25.00 & 1.23 & $5.92 \%$ \\
\hline $\mathbf{8}$ & 31.79 & 30.00 & 1.79 & $5.97 \%$ \\
\hline $\mathbf{9}$ & 36.92 & 35.00 & 2.92 & $5.48 \%$ \\
\hline $\mathbf{1 0}$ & 42.11 & 40.00 & 2.17 & $5.28 \%$ \\
\hline
\end{tabular}

As can be seen from Table 1, the method of the front of the traffic light distance measurement produced a certain error, the error causes include many factors, on the one hand, due to experimental conditions, the use of the left and right camera is a common CCD camera, precision Is not high; the other hand, the factors that produce experimental results may also be the camera calibration. According to the experimental results, the front traffic light distance measurement method based on binocular vision is suitable for the actual distance measurement method as the traffic light of the car safety driving system. Because the method has less than $6 \%$ error in the measurement of the traffic light in front and fully satisfies the safety of the vehicle System requirements of the actual range.

\section{Conclusion}

In this paper, the front traffic light distance measurement technology of binocular vision is analyzed and experimentally studied. The application of binocular stereoscopic vision technology in the range measurement of vehicle safety driving system is studied emphatically. Through the parallel binocular vision ranging principle, the measurement of the distance of the front traffic light is completed. The results show that the distance measurement method can meet the requirements of the front traffic light distance measurement. The design of the system is simple and fast, the measurement precision is high, the structure is easy to realize, and it has laid a good theoretical foundation for the intelligent and production of the vehicle 
safety driving system. The main research in this paper is to identify the traffic of the zebra and traffic lights with binocular vision technology for the traffic light junction. At the same time, the relevant information system integration and optimization to improve the measurement distance and accuracy, enhance the accuracy and real-time system. In order to further enhance the safety of the car driving function.

\section{Acknowledgements}

This work was supported by the National Science \& Technology Pillar Program during the 12th Fiveyear Plan Period (Grant No. 2015BAD18B01).

\section{References}

[1] Shen Lin. Research on high precision binocular CCD ranging[D].Changchun:Changchun University of Science and Technology, 2011:2-5.

[2] Zhang Zhengyou. Flexible camera calibration by Viewing a plane from unknown orierntations, International Conference on Computer Vision[C].Kerkyra:IEEE Xplore Digtal Library,1999.

[3] Ma Songde. A self-calibration technique for active vision system [J]. IEEE Transactions on Robot Automation, 1996, 12(1):114-120.

[4] Li Haijun, Xu Fengsheng, Zhang Jiancheng. Research on Application of Binocular Vision Automatic Recognition System [J], Journal of Changchun University of Science and Technology. 2007, 30(3):40-43.

[5] Li Xuejuan. Digital Range - finding Telescope Based on Binocular Parallax[D]. School of Precision Instrument and Optoelectronic Engineering, Tianjin University.2007:7-14.

[6] Liu Guoliang. Making and Research of Parallax Distance Measurement System Based on. Nanjing University of Posts and Telecommunications. 2013:14-19.

[7] Bu Fanyan. Research on Digital Matching Technology[D]. Hefei University of Technology2010: 34-41.

[8] Meng Yu, Fu Xianjie, Liu Li. A Method of Distance Measurement Based on Active Vision[J]. Journal of Central South University. 2013, 44(2):273-278. 